\title{
Vergleichende akustische Untersuchung von Drohnen-Propellern
}

\author{
Konrad Oeckel, Jan Heimann, Sven Angermann*, Michael Kerscher, Andreas Frahm, Gunnar Heilmann \\ und Wolfgang Rüther-Kindel
}

\section{Zusammenfassung}

Die Reduzierung von Fluglärm ist eine der wesentlichen Herausforderungen der Luftfahrtindustrie. Dies betrifft ebenso unbemannte Luftfahrtsysteme, die zukünftig eine signifikante Rolle im alltäglichen Leben spielen werden. In diesem Zusammenhang wurde im Fachgebiet Luftfahrttechnik der TH Wildau das energetische Potential von Winglets an Propellern („Proplets“) untersucht, deren Einsatz sowohl eine Verbesserung der aerodynamischen Effizienz als auch eine Minderung der Geräuschemission versprachen. Daher musste der akustische Einfluss dieser Proplets präzise bestimmt werden.

In diesem Beitrag wird erstmals die Geräuschemission eines mit Proplets ausgerüsteten Propellers mit einem Referenzpropeller verglichen. Beide Prüfstücke sind, abgesehen von den Blattspitzen, identisch. Neben normgerechten, herkömmlichen Messungen der Schallleistungspegel sowie psychoakustischer Eigenschaften werden beide Prüfstücke mit einem Mikrofon-Array („Akustische Kamera“) untersucht, um die Lärmquellen entlang der Propellerblätter zu visualisieren. Hierfür wird erstmals ein virtuell mitrotierendes Array angewendet, um die aero-akustischen Quellen am stehenden Blatt zu identifizieren.

Schlagworte: Fluglärm, Propellerlärm, Winglets, Proplets, Akustische Kamera, Schallquellenlokalisierung, rotational beamforming.

\section{Abstract}

The reduction of aircraft noise is one of the major challenges of the aviation industry. This also concerns unmanned aerial vehicles (UAVs), since they will play an increasingly important role in everyday life. In the context of the presented project the energetic potentials of winglets at propeller tips ("proplets") were investigated in order to increase the aerodynamic efficiency and decrease the noise emission of propellers. Therefore it was necessary to determine the acoustic effects of the attached proplets.

This paper compares the noise emission of a reference propeller with a proplet-equipped propeller for the first time. Except for the tips, they were identically designed. The acoustic power level of both propellers was determined and the results are assessed psycho-acoustically. Additionally an examination of the test pieces with a spaced microphone array ("Acoustic Camera") was conducted to visualize the localization of sound sources on the propellers. A rotational beamforming filter was applied to precisely identify the positions of the rotating aero-acoustic sources.

\section{Einführung und Ziel der Arbeit}

Die unbemannte Luftfahrt hat in den vergangenen Jahren einen beispiellosen Siegeszug angetreten. Im Jahre 2017 wurden weltweit bereits mehr als 3,5 Millionen UAVs (unmanned aerial vehicles - unbemannte Flugsysteme) verkauft, während auch industrielle Anwendungen wie Paketzustelldrohnen erprobt und eingesetzt werden. In diesem Zusammenhang konnte eine kürzlich veröffentliche Studie der
NASA allerdings ein signifikantes Ablehnungsverhalten der europäischen sowie US-amerikanischen Bevölkerung in Bezug auf das für UAVs typische, hochfrequente Fluggeräusch feststellen - auch im direkten Vergleich zu Straßenverkehrslärm.

Der Hauptanteil des Fluggeräusches, sowohl bei Multikoptern als auch bei konventionellen Tragflügeldrohnen, wird durch die Propeller emittiert. Aus diesem Grunde muss die akustische
Verbesserung an dieser Baugruppe ansetzen, um die allgemeine Akzeptanz für UAVs zu steigern.

In der jüngeren Vergangenheit wurden bereits einige Ansätze verfolgt, um den oben genannten Fluglärm zu mindern: Aufrauhen der Blattoberfläche, Ausfransen der Blatthinterkante oder die Nutzung von Proplets. Bis heute konnten allerdings die akustischen Potentiale dieser Ansätze nicht zweifelsfrei vermessen oder verglichen werden. 
In diesem wissenschaftlichen Beitrag wird nun zum ersten mal eine akustische Untersuchung umgesetzt, die einen mit Proplets ausgestatteten Propeller mit einem Referenzpropeller vergleicht. Mit Ausnahme der Blattspitzen weisen beide Propeller eine identische Geometrie auf. Sie wurden im Rahmen des Projektes SAPODS (Smart Airbourne Pollutants Detection System) im Fachgebiet Luftfahrttechnik der Technischen Hochschule Wildau gefertigt, um als Teil des Antriebssystems der fachgebietseigenen Messdrohne ATISS (Autonomous Testbed for Integrated Sensor Systems) Flughöhen von ca. 5.000 Meter zu erreichen.

In diesem Beitrag werden die akustischen Eigenschaften beider Propeller zunächst mit herkömmlichen Messmethoden untersucht. Hierzu gehört eine normgerechte Schallleistungsvermessung sowie eine psychoakustische Bewertung. Für einen umfassenderen Einblick in den Entstehungsmechanismus des Propellergeräusches zu gewinnen, ist es aber notwendig, die Schallquellen am Propellerblatt frequenzabhängig begutachten zu können. Aus diesem Grunde wird im Anschluss eine Untersuchung mit einer Akustischen Kamera vorgenommen. Dieses audiovisuelle Messsystem wurde im letzten Jahr um einen neuartigen Filter erweitert, mit dem nun die Schallentstehung von schnell rotierenden Objekten auf das stehende Objekt projiziert werden können. Somit kann nun erstmals eine präzise Begutachtung des Propellerlärms vorgenommen werden.

\section{Beschreibung der Prüfstücke}

Beide Propeller wurden von Mitarbeitern des Fachgebietes Luftfahrttechnik der TH Wildau konstruiert und gefertigt. Es handelt sich um Faltpropeller, die für eine Messdrohne mit einem maximalen Abfluggewicht von $250 \mathrm{~kg}$ entworfen wurden. Das gesamte Antriebssystem wurde für Flughöhen bis circa $5.000 \mathrm{~m}$ ausgelegt (Quaeck 2016). Alle geometrischen Kenngrößen sind jeweils identisch:
- Propellerdurchmesser: 22 / 558,8 mm

- Propellersteigung: 19“ / 482,6 mm

- Blattanzahl: 2

Beide Propeller wurden in Negativformbauweise als Nass-in-nass-Laminate aus kohlefaserverstärktem Kunststoff gefertigt. Die Negativformen verfügen über eine austauschbare Spitze, sodass die Profile entlang der Propellerblätter - und damit Kenngrößen, die den Gesamtschall des Propellers beeinflussen können, wie Blattdicke oder Wölbung - ebenfalls identisch sind. Die aerodynamische Effizienz der Propeller konnte durch verschiedene CFD-Testreihen überprüft werden (Angermann 2016).

Außerdem konnte Sven Angermann durch die Simulationsreihen die vielversprechendste Proplet-Konfiguration festlegen:

\section{- Typ: Blended Proplet}

nöhe: $30 \mathrm{~mm}$

Neigungswinkel: $0^{\circ}$

Krümmung: $0 \mathrm{~mm}$

Nachstehende Abb. 1 zeigt die 3D-Modelle beider für diesen Beitrag maßgeblichen Propeller.
Bei den oben beschriebenen Versuchen trat allerdings ein weiterer Effekt zu Tage: Von sämtlichen anwesenden Mitgliedern des Fachgebiets konnte ein wesentlich angenehmerer und weniger schneidender Klang des Propletpropellers festgestellt werden. Aus diesem Grunde sollen beide Propeller einer umfassenden akustischen Untersuchung unterzogen werden.

\section{Bestimmung der Schallleistungspegel}

Der erste Schritt einer vergleichenden akustischen Begutachtung ist die Bestimmung des Schallleistungspegels des Prüfobjekts, mithin die von sämtlichen Störeinflüssen bereinigte Schallenergie des Erzeugers. In diesem Beitrag wurde das Hüllflächenmessverfahren der Genauigkeitsklasse 2 nach DIN 3747:2011 angewendet (Deutsches Insitut für Normung 2011). Dieses Verfahren schreibt mindestens vier Messpositionen auf einer Halbkugel rund um das Prüfobjekt verteilt vor.

Bei der Vermessung der Propeller wurden fünf Messpositionen aufgezeichnet, die im Abstand von $40^{\circ}$ rund um den Propellerprüfstand platziert wurden. Damit konnte verhindert werden, dass sich die fünfte Messposition im Abwindfeld der Propeller befindet, wodurch die Messung aufgrund der auftretenden Luftmasseströme

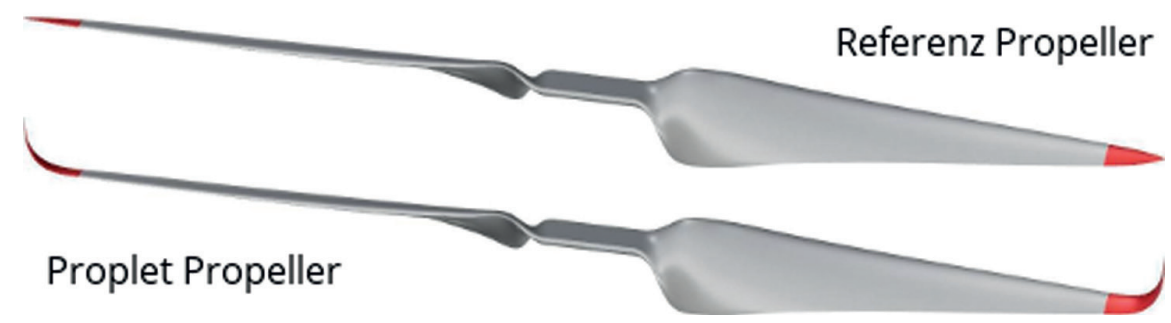

Abb. 1) Referenzpropeller und Propletpropeller (Angermann 2016).

Beide Propeller wurden auf dem Prüfstand für Elektroantriebe PRELA des Fachgebiets Luftfahrttechnik der TH Wildau untersucht, um die Simulationsergebnisse zu verifizieren. Entgegen der Hypothese, dass die Proplets einen positiven aerodynamischen Effekt bewirken, konnte bei diesen Prüfstandsversuchen ein geringfügig schlechterer Schubwirkungsgrad des Propletpropellers festgestellt werden. verfälscht worden wäre. Die Distanz zu den Propellern betrug jeweils 1,50 m. Abb. 2 zeigt das Schema des Messaufbaus zur Bestimmung der Schallleistungspegel. 


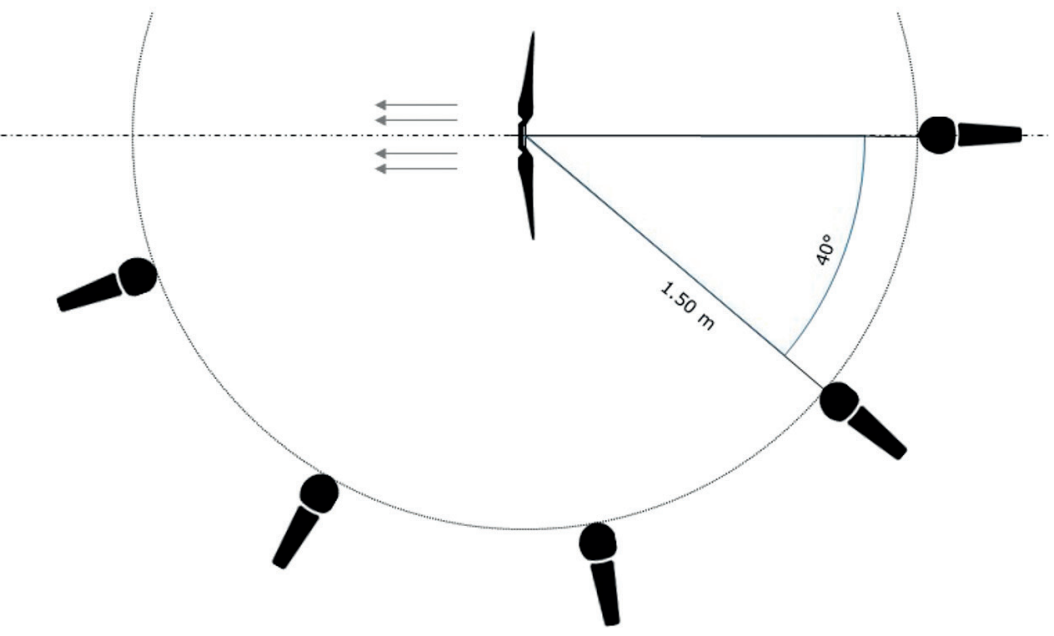

Abb. 2) Schematische Darstellung des Hüllflächenmessverfahrens.

Tab. 1) Ergebnisse der Bestimmung der Schallleistungspegel des Propellerpaares.

\begin{tabular}{|c|c|c|}
\hline Drehzahl & $\begin{array}{c}\text { Schallleistungspegel } \\
\text { Referenzpropeller }\end{array}$ & $\begin{array}{c}\text { Schalleistungspegel } \\
\text { Propletpropeller }\end{array}$ \\
\hline 2.500 min-1 & $92,5 \mathrm{~dB}$ & $93,3 \mathrm{~dB}$ \\
\hline $3.000 \mathrm{~min}-1$ & $94 \mathrm{~dB}$ & $96,1 \mathrm{~dB}$ \\
\hline $3.500 \mathrm{~min}-1$ & $97,6 \mathrm{~dB}$ & $98,1 \mathrm{~dB}$ \\
\hline $4.000 \mathrm{~min}-1$ & $100 \mathrm{~dB}$ & $101 \mathrm{~dB}$ \\
\hline $4.500 \mathrm{~min}-1$ & $102 \mathrm{~dB}$ & $104 \mathrm{~dB}$ \\
\hline
\end{tabular}

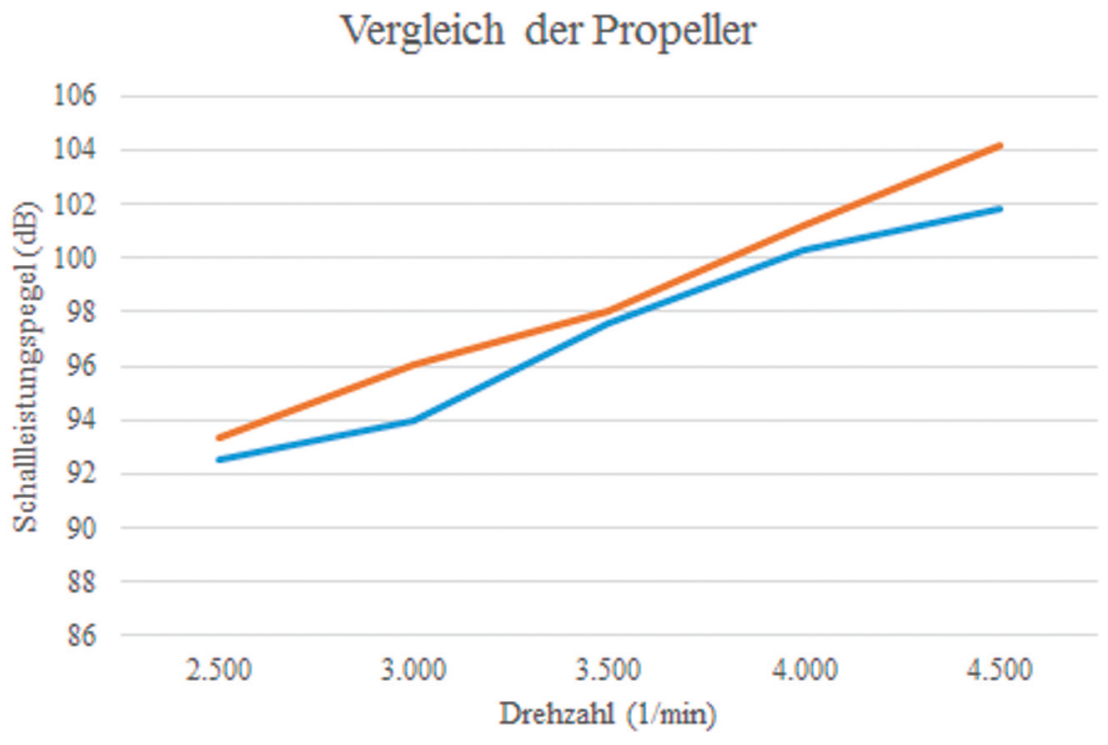

-reference propeller

Abb. 3) Vergleich der Schallleistungspegel beider Propeller.
Aus den an den Messpositionen aufgezeichneten Schalldruckpegeln konnten unter Berücksichtigung verschiedener in der Norm festgelegter Korrekturfaktoren für Umgebungsgeräusche oder Messraumcharakteristika die Schallleistungspegel für beide Propeller errechnet werden. Wie in nachstehender Tab. 1 zu sehen ist, wurde dieser Parameter über den kompletten Drehzahlbereich des Antriebssystems bestimmt.

Obwohl die Differenz beider Schallleistungspegel nicht mehr als etwa $2 \mathrm{~dB}$ pro Drehzahl beträgt, ist die Geräuschemission des Propletpropellers nachweislich etwas höher. Außerdem ist ein linearer Zusammenhang der errechneten Schallpegel ersichtlich (siehe Abb. 3). Der Arbeitsbereich des Antriebssystems wurde bei diesen Messungen fast vollständig abgedeckt, womit ausgeschlossen werden kann, dass sich die akustischen Eigenschaften des Propletpropellers in einem anderen Drehzahlbereich verändern.

Analog zu den Ergebnissen der aerodynamischen Prüfstandsversuche (Angermann 2016) kann also auch hier festgestellt werden, dass die Proplets einen negativen Einfluss auf die Gesamtschallerzeugung des Propellers haben.

\section{Psychoakustische Bewertung}

Eine Betrachtung der psychoakustischen Eigenschaften der Propeller verspricht Aufschluss über die subjektive Wahrnehmung der Propellergeräusche. Aus diesem Grunde wurden die Geräusche der Propeller bei den oben beschriebenen Versuchen über einen Zeitraum von jeweils 30 Sekunden aufgezeichnet. Somit ist es möglich den Frequenzverlauf der Propeller hinsichtlich des menschlichen Hörvermögens zu begutachten.

\subsection{Frequenzspektren}

Aus den Kurven gleicher Lautstärke DIN 226:2003 (Deutsches Institut für Normung 2003) geht hervor, dass der Mensch Schallereignisse im Bass- bzw. Hochtonbereich deutlich schlechter wahrnehmen kann, als im Mittentonbereich (siehe Abb. 4). Allerdings sinken die Verträglichkeits- 


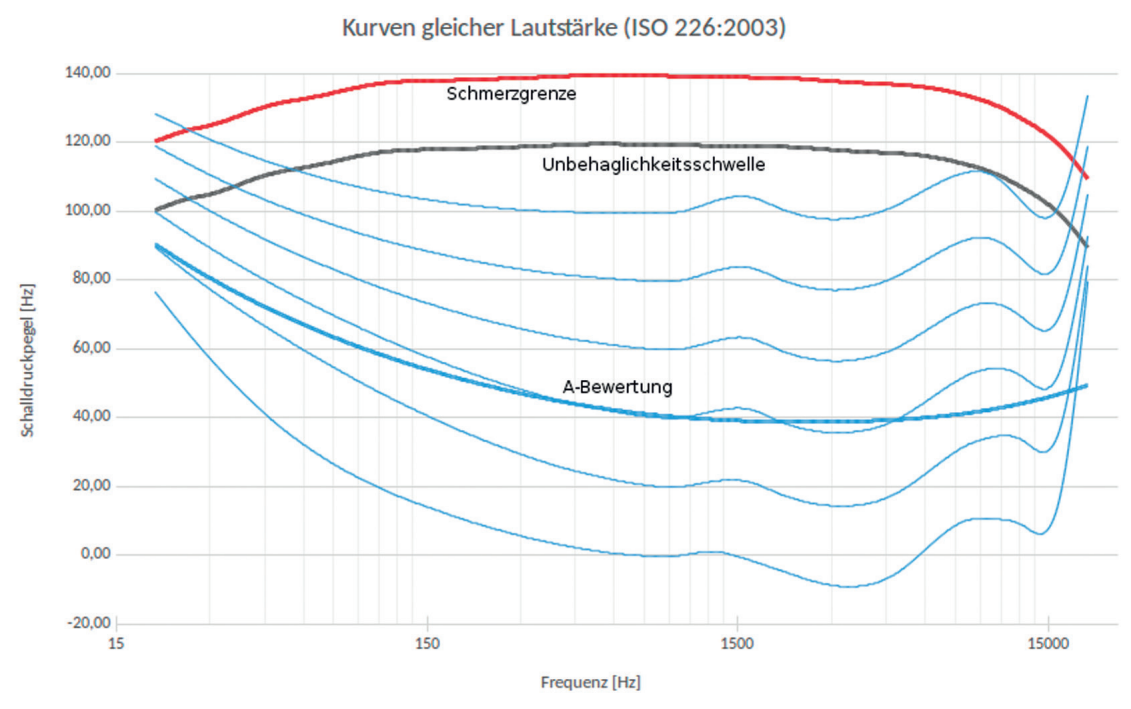

Abb. 4) Kurven gleicher Lautstärke mit Schmerz- und Unbehaglichkeitsschwelle.

schwellen in diesen Randbereichen deutlich schneller ab.

Die aufgezeichneten Geräusche der Propeller können durch eine schnelle Fouriertransformation in 1.024 Frequenzanteile zerlegt werden. Somit kann der jeweilige Frequenzgang dargestellt werden. In der nachstehenden Abb. 5 wurde eine lineare Skalierung der Abszisse gewählt, um vor allen Dingen den Hochtonbereich besser begutachten zu können.

Aus diesen Frequenzspektren geht hervor, dass der Propletpropeller deut-

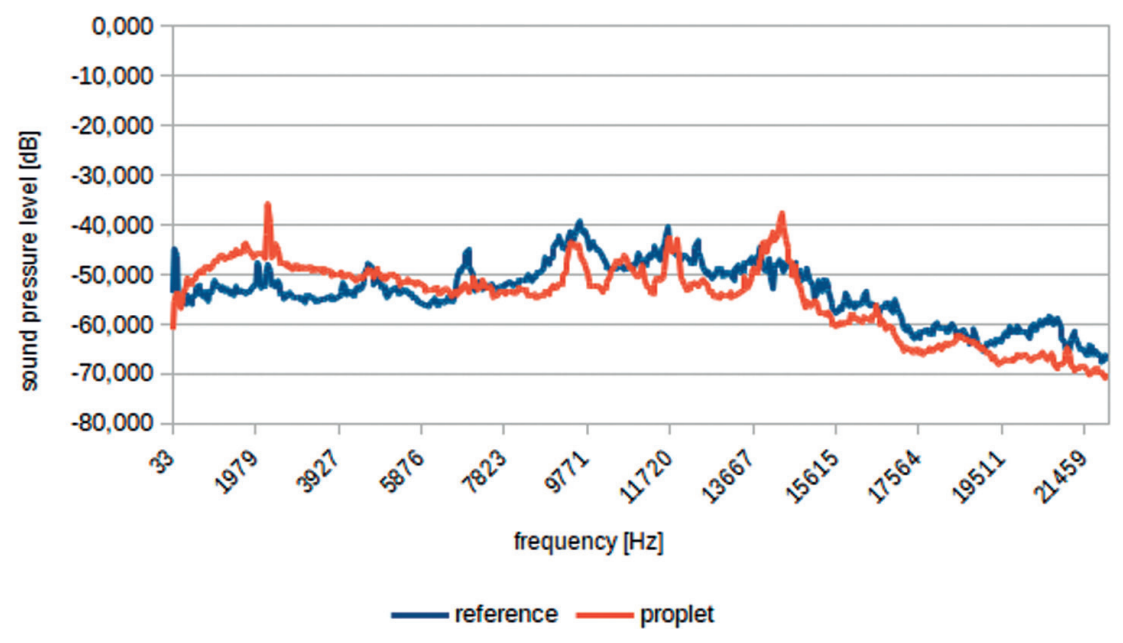

Abb. 5) Frequenzverläufe beider Propeller bei $3.000 \mathrm{~min}^{-1}$.

lich höhere Pegel im Mittentonbereich aufweist. Dies scheint in Bezug auf die Ergebnisse der Schallleistungsuntersuchung sinnvoll, da dieser Frequenzbereich den größten Anteil des Gesamtschallereignisses einnimmt. Im Hochtonbereich ab ca. $4.000 \mathrm{~Hz}$ ist

eine deutliche breitbandige Anhebung im Spektrum des Referenzpropellers sichtbar. Dieser Frequenzbereich ist mitbestimmend für die subjektive Wahrnehmung eines Schallereignisses, da die Unbehaglichkeitsschwelle in diesem Bereich deutlich absinkt und identische Schallpegel als deutlich störender empfunden werden, als bspw. im Mittenbereich.

\subsection{Schärfe}




\subsection{Prüfstand und Messraum}

Für die Untersuchung mit der Akustischen Kamera ist ein akustisch optimierter Messraum notwendig. Daher wurden die Messungen in der reflexionsarmen Schallkabine der Forschungsgruppe für Maschinendynamik und lärmarme Konstruktion der $\mathrm{TH}$ Wildau durchgeführt.

Des Weiteren wurde ein einfacher und mobiler Propellerprüfstand aufgebaut (siehe Abb. 6). Hierbei konnte eine Minimalkonfiguration genutzt werden, da keine weiteren aerodynamischen Parameter mit aufgenommen werden

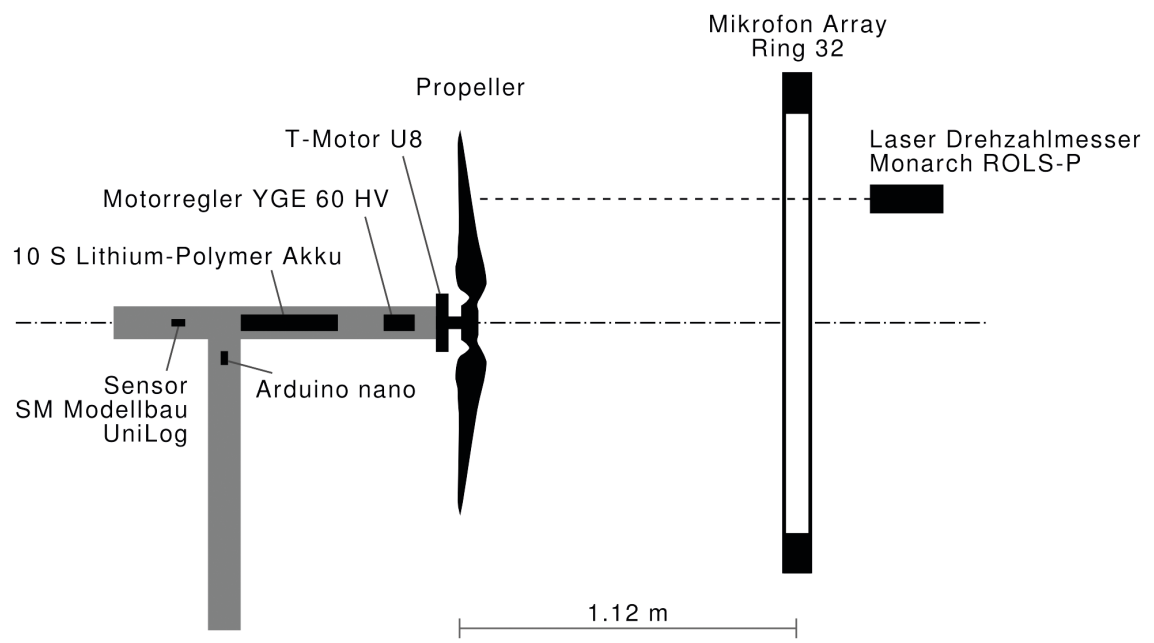

Abb. 6) Schematische Darstellung des Prüfstands.

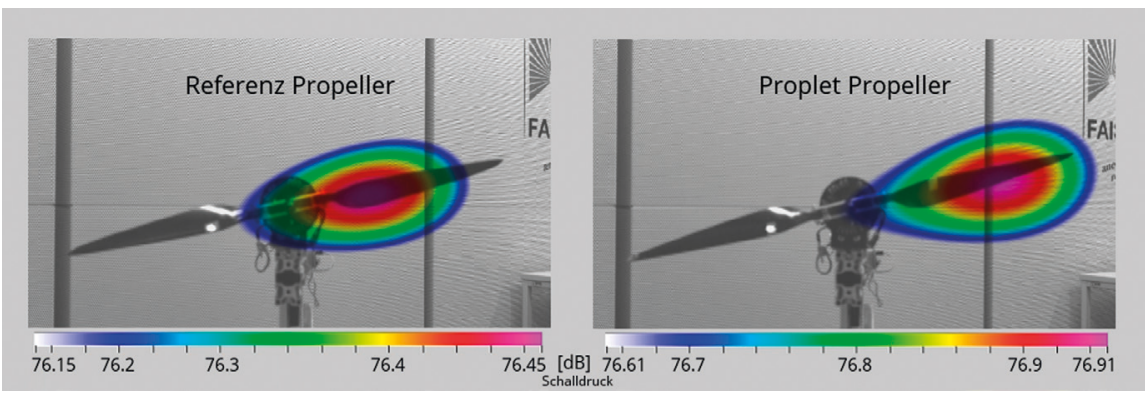

Abb. 7) Akustisches Foto beider Propeller - Terzband $1.600 \mathrm{~Hz}$.

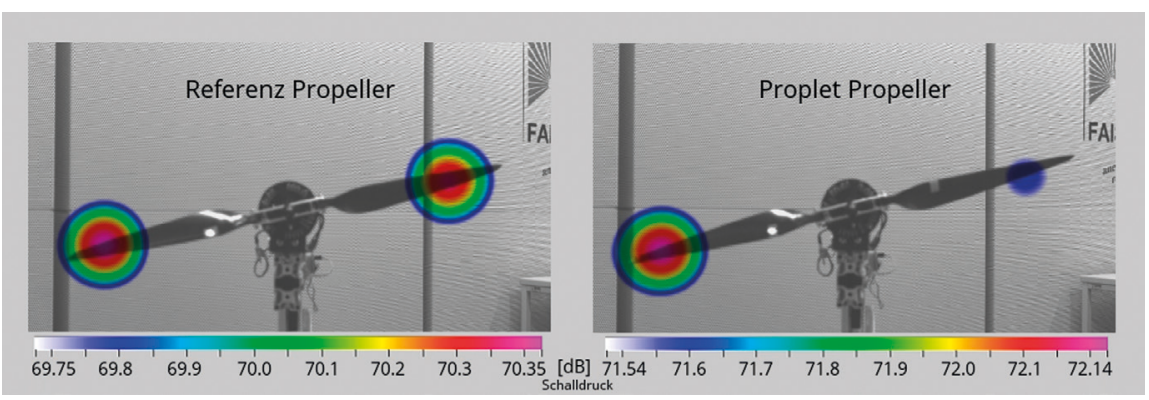

Laser-Drehzahlmesser genutzt, der direkt an das Messsystem angeschlossen werden kann. Aus diesem Grunde wurden Reflexionsstreifen auf den Propellern aufgebracht.

Nach dem Einstellen der Zieldrehzahl (3.000 $\mathrm{min}^{-1}$ ) wurden die Geräusche beider Propeller aufgezeichnet und mit einer Quantisierungstiefe von 24bit bzw. einer Samplerate von $96 \mathrm{kHz}$ gewandelt. Als Software sowohl für die Aufnahme als auch für die Analyse wurde das zum Messsystem gehörige Noiselmage 4.9 genutzt.

\subsection{Messergebnisse}

Nachdem der rotational beamformingFilter auf die aufgezeichneten Daten angewendet wurde, konnte nun ein Akustisches Foto des Propellergeräusches generiert werden. Hiermit können die Schallquellen entlang der Propellerblätter begutachtet werden. Um die Ergebnisse aus den Abschnitten 3 und 4 vertiefen zu können, wird im Folgenden eine Terzbandanalyse vorgenommen. Hiermit können die Schallquellen frequenzbasiert analysiert werden.

Abb. 7 zeigt das Mittenfrequenzband bei $1.600 \mathrm{~Hz}$.

Hier ist ein Einfluss der Propellerdicke auf die Schallemission zu sehen. Das Zentrum der Schallquelle befindet sich beim Referenzpropeller wie erwartet an der dicksten Stelle. Beim Propletpropeller befindet sich dieser Punkt allerdings weiter außen in Richtung der Blattspitze. Dies impliziert eine durch die Proplets induzierte, hochfrequente Blattschwingung und damit eine virtuelle Aufdickung des Profils. Die Aufnahme des Blattes am Mittelstück entwickelt keine Bremswirkung, da es sich um einen Falt- bzw. Klapppropeller handelt. Dieses Verhalten erklärt ebenso die in Abschnitt 2 genannten, schlechteren aerodynamischen Eigenschaften des Propletpropellers sowie den höheren Schallleistungspegel.

Weitere charakteristische Unterschiede sind im Terzband bei $3.000 \mathrm{~Hz}$ auszumachen (siehe Abb. 8). 
In diesem Akustischen Foto ist der Blattspitzenschlag zu sehen. Beim Referenzpropeller sind die Pegel der Schallquellen gleich verteilt. Beim Propletpropeller hingegen konzentriert sich die Schallenergie auf die linke Blattspitze. Das linke Blatt ist damit dominant. Grund hierfür könnte eine Unwucht sein, die durch eine Masseungleichheit der beiden Blätter (statische Unwucht) oder durch eine Verschiebung der Schwerpunktlagen (dynamische Unwucht) induziert ist.

In Abb. 9 ist das Schallverhalten der Propeller im Terzband bei $8.000 \mathrm{~Hz} z u$ sehen.

\subsection{Validierung}

Aus den Akustischen Fotos im vorherigen Abschnitt konnten verschiedene Annahmen hergeleitet werden, welche die unerwarteten Ergebnisse der aerodynamischen und akustischen Untersuchungen erklären können. Es ist allerdings notwendig, diese Thesen auf Sinnhaftigkeit zu prüfen.

Die vermutete Blattschwingung konnte durch eine Aufnahme mit einer Hochgeschwindigkeitskamera bestätigt werden. Hierbei wurde die Kamera senkrecht zur Propellerdrehebene platziert. Nach Einstellen der Zieldrehzahl

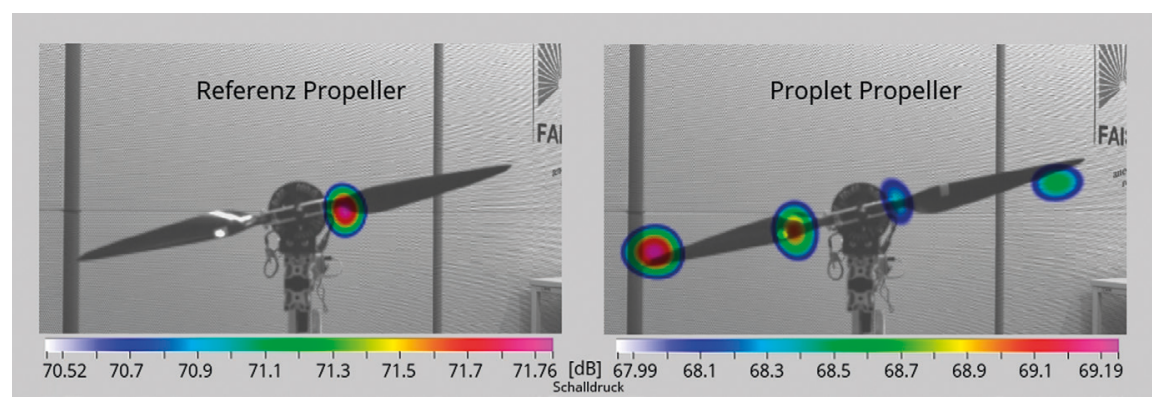

Abb. 9) Akustisches Foto beider Propeller - Terzband $8.000 \mathrm{~Hz}$.

Die Schallenergie des Referenzpropellers konzentriert sich hier auf einen Punkt an der Propellerwurzel. Dies weist auf einen Strömungsabriss hin, was plausibel erscheint, da der Anstellwinkel an dieser Stelle maximal ist. Das akustische Foto des Propletpropellers zeigt hingegen eine gleichmäßige Verteilung der Schallenergie, wobei auch hier das linke Blatt dominant erscheint.

Aus dieser Grafik wird ersichtlich, dass die Blätter des Propletpropellers mit deutlich größerer Amplitude oszillieren, als die des Referenzpropellers. Damit

Referenz Propeller Proplet Propeller
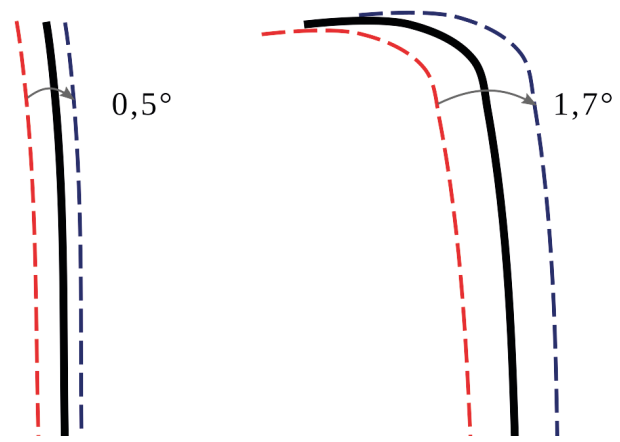

Mittelwert

Ausschlag min

Ausschlag max

Abb. 10) Blattauschläge von Referenz- und Propletpropeller. konnte die vermutete Blattschwingung nachgewiesen werden.

Weiterhin ist auf verschiedenen akustischen Fotos ein dominantes Blatt des Propletpropellers sichtbar geworden. Alle Propellerblätter wurden bei der Fertigung statisch gewuchtet, also auf ein Zehntel Gramm abgewogen worden. Allerdings wurde darauf verzichtet, den Schwerpunkt der Blätter zu bestimmen, mithin eine dynamische Wuchtung vorzunehmen. Bei der Überprüfung des Propletpropellers konnte nun festgestellt werden, dass sich die Schwerpunktlage entlang der Propellersehne um 0,91 mm unterscheidet. Beim Referenzpropeller beträgt diese Differenz lediglich 0,13 mm. Demnach konnte auch die vermutete (dynamische) Unwucht nachgewiesen werden.

\section{Fazit}

In diesem Beitrag konnte eine umfängliche Messmethode erarbeitet werden, die es ermöglicht, die Lärmemission von Propellern besser zu verstehen und präziser zu analysieren. Fertigungsmängel konnten identifiziert werden. Durch die Anwendung des rotational beamforming-Filters ist die Akustische Kamera nun in der Lage, einen tieferen Einblick in das Schallverhalten am Propellerblatt zu gewähren und lärmmindernde Potentiale künftig zielgerichteter anzuwenden. Somit können auch die bisher in der Fachliteratur verfügbaren, meist theoretischen Ansätze der Propellerlärmanalyse vertieft und erweitert werden.

Weiterhin konnte gezeigt werden, dass der Einsatz von Proplets einen Einfluss auf die Geräuschentwicklung der Propeller hat. Sie können ein Ansatz darstellen, um künftig die Akzeptanz von UAVs zu steigern, da sie die Schärfe des Propellerlärms abmildern, wodurch der Klang des Propellers als angenehmer bzw. weniger störend wahrgenommen wird.

Es wird daher empfohlen, die akustischen Potentiale der Proplets noch umfangreicher zu analysieren. Hierzu könnten die oben beschriebenen Messungen mit festgestellten Propellerblättern sowie mit Proplets 
halber Höhe durchgeführt werden. Außerdem sollte die Messmethode auf größere Antriebssysteme umgesetzt werden, da hier aufgrund der steigenden Anzahl von Propellermaschinen im Sportluftverkehr sowie im Kurzstreckenlinienverkehr noch größere Notwendigkeiten der Lärmvermeidung angezeigt sind.

\section{LITERATUR}

Angermann S (2016) Investigations of Propeller Optimization by using Proplets. Dissertation, Universita degli studi di Roma "Tor Vergata“"

Deutsches Institut für Normung (2003) ISO 226:2006-04. Acoustics - Normal equal-loudness-level contours, Berlin

Deutsches Insitut für Normung (2009) DIN 45692:200908 . Measurement technique for the simulation of the auditory sensation of sharpness, Beuth

Deutsches Institut für Normung (2011) DIN EN ISO 3747:2011-03. Acoustics - Determination of sound power levels and sound energy levels of noise sources using sound pressure - Engineering/survey methods for use in situ in a reverberant environment, Beuth

Kerscher M, Heilmann G, Puhle C, Friebe C, Krause R (2017) Sound Source Localization on a Fast Rotating Fan Using Rotational Beamforming. In: InterNoise. $46^{\text {th }}$ International Congress and Exposition on Noise Control Engineering, 27.-30.08.2017, Hong Kong

Quaeck F (2016) Auslegung eines höhentauglichen Antriebes für das unbemannte Luftfahrtsystem ATISS. Masterarbeit, Technische Hochschule Wildau

\section{AUTOREN}

Dr.-Ing. Sven Angermann

Dr. Andreas Frahm

Konrad Oeckel, B. Eng.

Prof. Dr.-Ing. Wolfgang Rüther-Kindel

Fachgebiet Luftfahrttechnik

Luftfahrttechnik / Luftfahrtlogistik

Technische Hochschule Wildau

Gunnar Heilmann

Jan Heimann

Michael Kerscher

gfai tech $\mathrm{GmbH}$

E-Mail für Korrespondenz:

sven.angermann@th-wildau.de 